\title{
Latest ERS Guidelines on non-CPAP Therapies in Obstructive Sleep Apnoea
}

Heeral Patel

Editorial Assistant

Citation: EMJ Respir. 2021;9[1]:28-30.
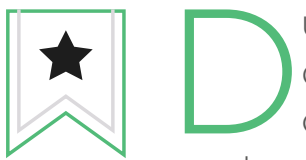
uring the European Respiratory Society (ERS) International Congress 2021, speakers discussed the latest updates to the ERS guidelines on non-CPAP therapies in obstructive sleep apnoea. The new recommendations were drawn following a systemic review of the literature.

OBSTRUCTIVE sleep apnoea (OSA) is the most common sleep breathing disorder, affecting up to 100 million individuals around the globe. Patients with OSA have several episodes throughout the night where they struggle to breathe due to the collapse of their upper airways during sleep. Some of the most common symptoms of OSA include fatigue, daytime sleepiness, and disruptive snoring. OSA is a chronic disease with no cure and requires careful phenotyping and constant patient engagement with therapy.

Continuous positive airway pressure (CPAP) therapy is often the first line of treatment for OSA, which involves a mask or nose piece that delivers positive air pressure to the airways, forcing the airways to stay open. Although this is an effective treatment, many patients have reported discomfort sleeping with a mask. This ERS guidelines session hosted a discussion of the recent updates to the guidelines on non-CPAP therapies in OSA, determined by a systemic review of the literature.
The first speaker, Sophia E Schiza, Head of the Sleep Department, University of Crete, Greece, highlighted the gaps in OSA treatment as well as previous and current clinical practice. She explained how the refinement of techniques has led to an update on the ERS guidelines on nonCPAP therapies in OSA. Until 2012, the Chicago criteria were used to calculate the apnoeahypopnea index $(\mathrm{AHI})$, which is the number of apnoeas or hypopneas recorded per hour of sleep in a study. Despite $A H I$ being a helpful metric in defining the presence of OSA, the speaker acknowledged that $\mathrm{AHI}$ has its limitations.

Firstly, the calculation of AHI assumes apnoeas and hypopneas are equal in their biological effects. Secondly, AHI does not indicate the magnitude of oxygen desaturation. In addition, $\mathrm{AHI}$ does not account for gender or age-related changes. In other words, two patients with OSA with similar $\mathrm{AHI}$ scores could have significantly different severity of OSA, depending on other factors such as age, occupation, and associated conditions. The use of $\mathrm{AHI}$ as a continuous exposure variable is based on the assumption that 


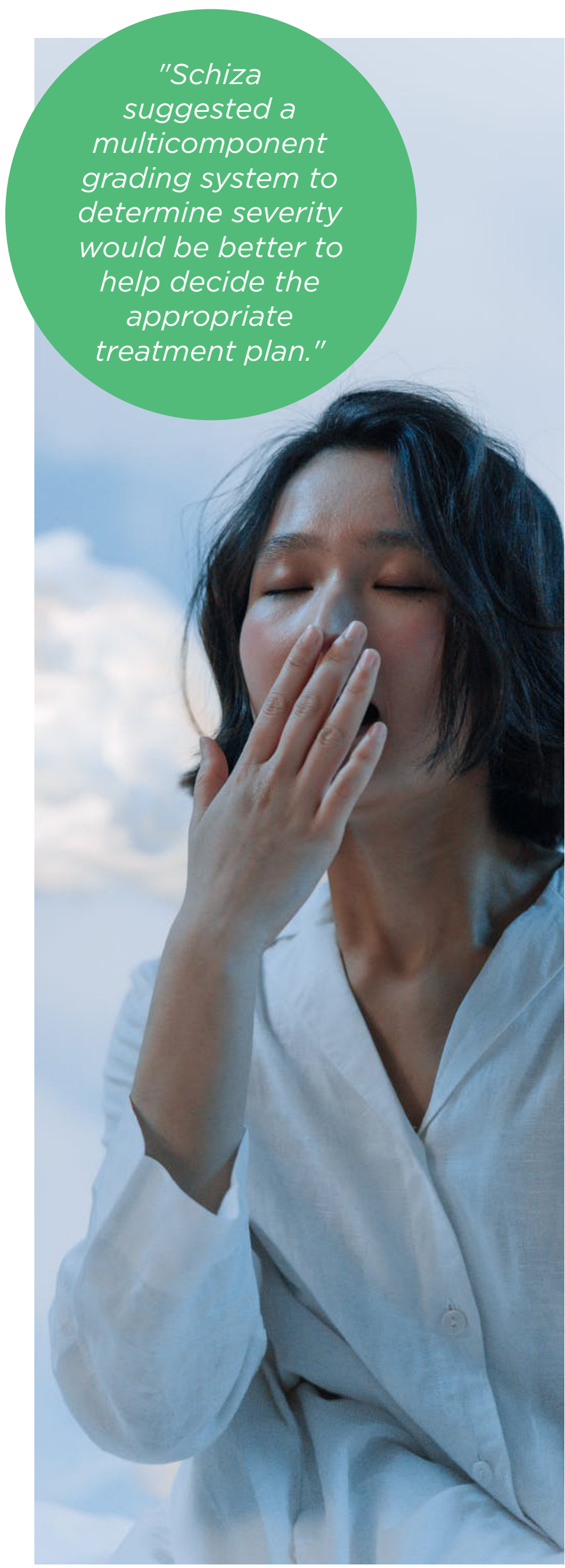

it represents the disease state of OSA. However, this assumption has proven to be incorrect in the literature several times. Based on this conclusion, using $\mathrm{AHI}$ as the primary diagnostic tool of clinically relevant OSA should be reconsidered. Schiza suggested a multicomponent grading system to determine severity would be better to help decide the appropriate treatment plan.

Following the first speaker, Johan Verbraecken, University of Antwerp, Belgium, explained the recommendations on ERS guidelines on OSA in further detail as an update to the 2011 ERS guideline on non-CPAP therapies in OSA. The updated guidelines indicate that treatment recommendations must be based on different types of evidence, clinical experience, and other relevant factors. The speaker shared the various and novel interventions available for OSA, including gastric bypass surgery, custom-made duo-block mandibular advancement devices, hypoglossal nerve stimulation, myofunctional therapy, acetazolamide, and positional therapy. To end, Verbraecken presented a case study of a 57-year-old man who did not want CPAP. Using the latest guidelines, he concluded that myofunctional therapy would be the best solution in this individual scenario.

The final speaker, Joerg Steier, Guy's and St Thomas' Hospital, London, UK, discussed a few interesting cases that he had encountered in his sleep clinic. Steier acknowledged that CPAP is an effective treatment option for patients and addressed the critical question: what is the point of discussing non-CPAP therapies? As previously mentioned, some patients do not like wearing the mask and compliance is low. In fact, compliance can be as low as 50\% in younger cohorts of patients and even less in females. Therefore, it is important to discuss non-CPAP therapies. The new guideline addresses different interventions such as gastric bypass surgery, carbonic anhydrase inhibitors, and positional therapy to help patients manage their OSA.

The first case Steier presented was a 54-yearold male who was referred to the sleep centre in 2007 due to excessive daytime sleepiness. The physicians ran some tests and measured the desaturation of oxygen during sleep. The patient was diagnosed with severe OSA and, consequently, they were referred to sleep apnoea therapy. At first, CPAP controlled the problem 
